\title{
Empresas en la cooperación gubernamental española 2009-2012
}

\section{RESUMEN}

Elisa Pintado Maroto elisapintado@gmail.com Universidad de Valladolid, Valladolid, España.

\begin{abstract}
La base de la investigación es una base de datos realizada fundamentalmente con la información proporcionada por el ministerio de Asuntos Exteriores sobre la Ayuda Oficial al Desarrollo (AOD) española durante los años 2009-2012. En esa base de datos se ha recopilado exclusivamente la información referida a empresas en un sentido amplio del término que hayan recibido dinero público considerado AOD para realizar proyectos de cooperación. Con esa información se están extrapolando datos estadísticos para tener una imagen clara de cuál ha sido el papel del sector empresarial en la cooperación gubernamental para el desarrollo durante la vigencia del último Plan Director de la Cooperación Española 2009-2012, analizando cuantías, orientación geográfica, orientación sectorial y tipología de las empresas, entre otros.
\end{abstract}

PALABRAS CLAVE: Cooperación empresarial. RSC. Responsabilidad Social Corporativa. AOD. Ayuda Oficial al Desarrollo. 


\section{INTRODUCCION}

La cooperación internacional para el desarrollo y el sector empresarial son dos campos que cada vez se vinculan con mayor frecuencia. La Responsabilidad Social Corporativa (RSC) o Responsabilidad Social Empresarial (RSE) son conceptos que se manejan cotidianamente por las empresas en la actualidad. Tanto desde la cooperación gubernamental como desde las ONGD ha aumentado la participación con el sector empresarial y cada vez cuentan más con ellos a la hora de llevar a cabo sus proyectos o actividades de desarrollo. La normativa internacional y nacional de cooperación permite considerar a las empresas privadas como agentes de cooperación más allá de su rol tradicional de proveedor de bienes y servicios.

A pesar de las críticas y apoyos que provoca la aparición de este nuevo agente y de los enormes esfuerzos por introducirlo por parte de las distintas administraciones, en realidad no se sabe muy bien cuál es su peso real en la AOD, qué empresas participan, qué tipos de proyectos realizan o en qué áreas y sectores. Por eso resulta interesante la posibilidad de realizar una fotografía a la cooperación española para ver en qué punto estamos y cuál es la tendencia real más allá de las intenciones políticas. Ese es el objetivo fundamental de esta investigación.

\section{CONSIDERACIONES PREVIAS}

Desde que en el año 1999 Kofi Annan presentara su propuesta de lo que luego se conocería como Pacto Mundial o Global Compact, han pasado 15 años en los que se ha intentado argumentar cuál sería la justificación de la introducción del sector privado en la lucha activa contra la pobreza para alcanzar los distintos objetivos del desarrollo: Declaración de Doha, Declaración de París, Programa de acción de Accra o más recientemente la Declaración de Busán.

Para el caso español, además de unirse a todos estos planteamientos a través de los últimos Planes Directores y otras declaraciones como la "Estrategia de crecimiento económico y tejido empresarial (MAEC, SECl, 2011), realizada desde la Secretaría de Estado de Cooperación, o la última modificación de la ley que crecó el FONPRODE (Fondo de Promoción del Desarrollo) - Ley 36/2010, de 22 de octubre -, lo cierto es que la propia ley de cooperación española de 1998 (Ley 23/1998, de 7 de julio, de Cooperación Internacional para el Desarrollo) ya hace una referencia explícita sobre el papel que cumplirían las empresas como agentes de cooperación, en donde se las legitima como tales, pudiendo participar en todo tipo de actividades de cooperación y poniendo especial énfasis en su papel en la cooperación técnica y que éstas podrán firmar convenios, solicitar subvenciones o establecer otras formas de relación con las distintas administraciones. Sin embargo, hay que remarcar que en la propia ley se indica, de manera muy explícita, que estas mismas administraciones deberán garantizar que estos proyectos en los que participen las empresas no pueden tener ningún carácter lucrativo. 
Viendo que la participación de la empresa en la cooperación está legitimada por la propia ley de cooperación, lo siguiente sería ver cómo admite la cooperación descentralizada la participación de empresas. Desde que en el año 1999 se crea la primera ley autonómica de cooperación, la de la Comunidad de Madrid, se inicia una carrera entre las distintas comunidades autónomas por proveerse de una ley de cooperación específica que regule las características propias de la cooperación al desarrollo de cada una de las regiones españolas. De las 17 leyes de cooperación de las comunidades autónomas, 12 consideran a las organizaciones empresariales como agentes de cooperación y 9 a las propias empresas como agentes (algunas leyes reconocen a ambos tipos de entidad). $\mathrm{Ni}$ Asturias ni País Vasco reconocen de manera explícita a las empresas como agentes de cooperación, aunque sí a entidades sin ánimo de lucro en donde podrían estar incluidas las organizaciones empresariales.

El caso de los municipios es mucho más complicado. La mayoría actúa bajo la ley de cooperación de la comunidad autónoma donde se encuentren, aunque en algunos casos nos podemos encontrar con planes de cooperación municipales, aunque son escasos y no suelen comentar cuestiones de empresas.

Teniendo en cuenta todos estos datos se puede afirmar que las empresas están legitimadas para poder ejercer como agentes de cooperación y por lo tanto poder acudir a convocatorias de subvenciones.

\section{METODOLOGÍA}

\section{DELIMITACIÓN DEL OBJETO DE ESTUDIO}

Esta investigación se ha centrado en la cooperación gubernamental realizada entre los años 2009 y 2012, los años de vigencia del ya finalizado Plan Director de la Cooperación Española 2009-2012, ya que resultaba más interesante el presentar un análisis de un periodo ya concluido de la cooperación española. Sin embargo no resultaba útil analizar todos los datos de todas las administraciones que realizan cooperación por lo que se procedió a realizar un muestreo de cuáles eran las entidades qué más dedicaban a cooperación al desarrollo descartando aquellas que lo hacían en menor cuantía o de manera más marginal. En el momento de inicio de este estudio no estaban disponibles muchos de los datos, de manera que la selección se hizo de acuerdo a la información presente en aquel momento, los datos de los años 2009 y 2010. Aunque estaba claro que sí se iban a analizar todos los datos de la administración central, este primer análisis ayudó en la elección de comunidades autónomas y ayuntamientos. Teniendo en cuenta el volumen de AOD de cada administración, la elección que se hizo fue la siguiente:

Tabla 1 - Entidades seleccionadas para el estudio

\begin{tabular}{|c|c|}
\hline Comunidades Autónomas & Ayuntamientos \\
\hline Andalucía & Madrid \\
\hline Cataluña & Barcelona \\
\hline País Vasco & Zaragoza \\
\hline Comunidad Valenciana & Bilbao \\
\hline Madrid & Vitoria \\
\hline
\end{tabular}




\begin{tabular}{|c|c|}
\hline Navarra & Córdoba \\
\hline Canarias & Pamplona \\
\hline & Palma de Mallorca \\
\hline & Burgos \\
\hline & Gijón \\
\hline & Logroño \\
\hline
\end{tabular}

Fuente: elaboración propia

En lo que respecta a la cooperación central, se ha analizado toda la presentada por los diferentes ministerios, con especial énfasis en la referida al Ministerio de Asuntos Exteriores y Cooperación y al Ministerio de Economía.

\section{FUENTES, PROBLEMAS Y OTRAS CONSIDERACIONES:}

Los datos de la cooperación gubernamental para el desarrollo son públicos ya que todos los años desde el Ministerio de Asuntos Exteriores y Cooperación se hacen públicos lo que llaman Seguimiento del PACl -siglas de Plan Anual de Cooperación Internacional-. Consiste fundamentalmente en un informe en el que se realiza un análisis de toda la AOD española -incluyendo la cooperación de la administración central, la cooperación descentralizada y la cooperación universitaria- y en un volcado de datos en formato Excel donde se han introducido todas las acciones de cooperación realizadas en dicho año con una descripción razonablemente detallada: quién realizó la acción, en qué consistía, desembolso económico, orientación geográfica y sectorial, etc. También se incluyen los datos referentes a la tipología de la entidad que canaliza los fondos de una acción, incluyendo empresas privadas, si es ayuda ligada, si existe cofinanciación privada y otros que son fundamentales para analizar la cantidad de AOD que es canalizada a través de empresas. Además también se puede ver cuál fue el mecanismo de concesión de dicha subvención o qué unidad específica concedió los fondos. Estos datos proporcionan una información muy valiosa, de manera que fue la fuente fundamental para la realización de esta investigación.

Sin embargo todos estos datos no son perfectos. Los datos son introducidos por los responsables y técnicos de cooperación de las distintas administraciones y en ocasiones contienen errores, inexactitudes y vacíos de información. Para intentar compensarlo se decidió realizar una nueva base de datos solamente con los datos referentes a empresas para que fuera más manejable y que pudiera arrojar información sin estar entorpecida por datos no interesantes para este estudio y con correcciones respecto a la original para intentar ver una fotografía más clara del objeto del estudio. De hecho los dos principales problemas que se nos presentaron a la hora de realizar la base de datos solamente con la información referida al dinero público transferido a empresas para realizar proyectos de cooperación fueron la mala clasificación de las entidades canalizadoras y el vacío de información.

En lo que respecta al primero de los problemas, lo que ocurre es que en ocasiones las empresas no son clasificadas como empresas, sino que son clasificadas como cualquier otra cosa, entidades públicas, ONGD, "otras entidades", etc. Con lo cual, la búsqueda de empresas entre las beneficiarias de subvenciones públicas se complica muchísimo al no poder limitarse a la clasificación hecha por el ministerio. La única solución posible es la revisión una a 
una de todas las entidades receptoras. Esto es muy laborioso ya que de media cada año hay unas 35.000 acciones para revisar, más de 100.000 entradas de acciones diferentes para los cuatro años, lo que lleva muchísimo tiempo ya que hay que revisarlas una a una. En otras ocasiones, el problema no es que la clasificación esté mal, sino que el nombre de la entidad introducida puede contener erratas, lo que sucede en muchas ocasiones sobre todo cuando en vez de nombres completos de entidades se introducen siglas. O también nos encontramos con entidades de las que no se ha localizado ninguna información o la información encontrada no explica qué tipo de entidad son.

En lo que respecta a los vacíos de información, ésta está siendo rellenada a través de las fuentes alternativas de la cooperación, fundamentalmente las resoluciones oficiales de proyectos publicados en los diferentes boletines oficiales, memorias de cooperación de las administraciones, cuestionando a los técnicos y responsables de las diferentes entidades, etc.

En cualquier caso es conveniente resaltar que la información presentada en este artículo no está todavía consolidada. Debido al volumen de información los vacíos que ésta presenta son muy lentos de rellenar y la información requerida del año 2012 ha sido publicada en diciembre de 2013, con lo cual todavía queda información por procesar. A pesar de esto, ya es posible extraer conclusiones tanto de la información de la que disponemos como de la que no disponemos.

\section{DATOS DE AOD Y EMPRESAS}

Para dar una visión general sobre qué fondos públicos se están transfiriendo a empresas como parte de las políticas de cooperación es interesante ver la relación entre el total de los fondos y los dedicados específicamente para empresas como agentes. La cantidad de fondos dedicados a empresas ha sido muy variable en estos cuatro años de estudio, alcanzando el clímax en el año 2010, el primer año de funcionamiento del Fondo de Promoción del Desarrollo. Ese año, de los 3.900 millones de euros dedicados a cooperación por las administraciones centrales, más de 450 millones pasaron a entidades con ánimo de lucro, algo más del $11 \%$ del total. Es algo excepcional, puesto que en el año 2009 y en los años 2011 y 2012 la cantidad no superó en ningún caso los 50 millones.

Tabla 2 - AOD por entidades y empresas

\begin{tabular}{|c|c|c|c|c|c|c|c|c|}
\hline & $\begin{array}{c}\text { AOD (mill. } \\
€)\end{array}$ & $\begin{array}{c}\text { AOD } \\
\text { empresas } \\
\text { (mill. €) }\end{array}$ & $\begin{array}{c}\text { AOD } \\
\text { (mill. €) }\end{array}$ & $\begin{array}{c}\text { AOD } \\
\text { empresas } \\
\text { (mill. €) }\end{array}$ & $\begin{array}{c}\text { AOD (mill. } \\
€)\end{array}$ & $\begin{array}{c}\text { AOD } \\
\text { empresas } \\
\text { (mill. €) }\end{array}$ & $\begin{array}{c}\text { AOD } \\
\text { (mill. €) }\end{array}$ & $\begin{array}{c}\text { AOD } \\
\text { empresas } \\
\text { (mill. €) }\end{array}$ \\
\hline & \multicolumn{2}{|c|}{2009} & \multicolumn{2}{|c|}{2010} & \multicolumn{2}{|c|}{2011} & \multicolumn{2}{|c|}{2012} \\
\hline Municipios & 64,8 & 2,6 & 57,5 & 1,5 & 31,3 & 0,3 & 11,6 & 0,5 \\
\hline $\begin{array}{c}\text { Comunidades } \\
\text { Autónomas }\end{array}$ & 325 & 9 & 283 & 9,25 & 162 & 1,5 & 142,5 & 2 \\
\hline AGE & 4.128 & 37 & 3.969 & 454 & 2.687 & 39 & 1.365 & 40 \\
\hline
\end{tabular}

Fuente: Elaboración propia

Todos estos datos son todavía provisionales a la espera de poder analizar todos los datos y ausencias de datos proporcionados por las administraciones públicas. En municípios, se ha contabilizado la AOD de los municipios y 
comunidades autónomas objeto de estudio, no están incluidos el resto de entidades que no se hayan estudiado

Es llamativo como el porcentaje de AOD destinado a empresas no ha aumentado con el tiempo, sino más bien todo lo contrario, y con excepción de los datos del año 2010, para el resto de años la cantidad es bastante pequeña en proporción, aunque siempre son de decenas de millones de euros y en una cantidad bastante constante, a pesar de que las cifras de AOD han descendido drásticamente.

Para las comunidades autónomas la cantidad es pequeña en comparación del total y desciende en los años 2011 y 2012 de manera llamativa. En cualquier caso, en el año 2010 se llegaron a destinar para empresas más de 9 millones de euros, aunque el porcentaje no llega en este caso al $4 \%$ del total de la AOD de las autonomías. Para los dos últimos años, el porcentaje no llega apenas al $1 \%$ del total. Tiene sentido que los porcentajes de fondos destinados a empresas sean menores en las comunidades autónomas que en las administraciones centrales, debido a que fundamentalmente su único instrumento de cooperación suele ser la convocatoria de subvenciones, aunque muchas de ellas también firman convenios. También por los principios de la cooperación descentralizada, que suele, en general, fomentar más la participación de las ONGD de cada comunidad.

Para los ayuntamientos las cifras son más altas de lo que a priori cabría esperar. En el año 2009 se llegaron a dedicar 2,6 millones de euros, un 4\% del total. Viendo el descenso radical en los años posteriores de la cooperación de los ayuntamientos estudiados no es sorprendente ver que también reduzcan las cifras dedicadas a empresas. Sin embargo, en el año 2012, con unas cifras mínimas en la cooperación municipal, la cifra dedicada a empresas se mantiene al $4 \%$, las mismas cifras que en el año 2009 y aumentando los porcentajes de los años anteriores.

En lo que respecta a la orientación geográfica de todos estos fondos, lo más útil es analizar los de la cooperación de la administración general del estado puesto que la concentración geográfica es mayor y siguen directrices comunes, a diferencia de los ayuntamientos y comunidades autónomas, donde cada una tiene sus propias prioridades.

Tabla 3 - Orientación geográfica de la AOD de la AGE canalizada por empresas (mill. €)

\begin{tabular}{|c|c|c|c|c|} 
& África & América & Asia & Europa \\
\hline AGE 2012 & 19 & 8 & 13 & \\
\hline AGE 2011 & 9,1 & 12,6 & 10,5 & \\
\hline AGE 2010 & 274 & 54,3 & 52,3 & 58 \\
\hline AGE 2009 & 5,7 & 0,8 & 11 & 2 \\
\hline
\end{tabular}

Fuente: elaboración propia

Mientras que en el año 2009 la mayoría de los fondos estaban orientados hacia Ásia, mientras que la AOD orientada hacia el continente americano era puramente testimonial -recibió menos fondos que los dedicados a países europeos- esta tendencia cambia drásticamente en el año 2010, donde la gran mayoría de fondos son redirigidos hacia África, fundamentalmente África Subsahariana. Esta tendencia vuelve a repetirse en el año 2012, donde África 
vuelve a ser el principal destino tras el año 2011, cuando el continente americano fue el más beneficiado. Llama la atención que mientras en los años 2011 y 2012 Europa no está representada en la orientación de fondos, en el año 2010 fue el segundo destino de los mismos. Fundamentalmente éstos se dirigieron hacia Turquía y los países balcánicos.

Tabla 4 - Tipología de la ayuda de la AOD destinada a empresas (mill. €)

\begin{tabular}{|c|c|c|c|c|}
\hline & $\begin{array}{c}\text { Ayuda } \\
\text { programática }\end{array}$ & $\begin{array}{c}\text { Cooperación } \\
\text { Técnica }\end{array}$ & $\begin{array}{c}\text { Proyectos de } \\
\text { Inversión }\end{array}$ & Sin datos \\
\hline AGE 2012 & & 4,3 & & 35 \\
\hline AGE 2011 & 12,5 & 0,03 & 0,1 & 26,2 \\
\hline AGE 2010 & 6,8 & 12,4 & 413 & 22,7 \\
\hline AGE 2009 & 0,6 & 0,034 & & 36 \\
\hline
\end{tabular}

Fuente: elaboración propia

Lo primero que llama la atención al ver la tabla dedicada a la tipología de la ayuda es que para los años que no son el 2010 faltan proporcionalmente muchos datos, así como las ínfimas cantidades que se dedican a cooperación técnica y ayuda programática. Esto hay que mantenerlo un poco en reserva hasta que se puedan analizar todos los datos, ya que la persona que los introdujo en las bases de datos oficiales puede que desconociera el cariz de esa ayuda. Es decir, puede que se dediquen más fondos a cooperación técnica, pero todavía no lo sabemos. En cualquier caso, lo que sí sabemos es que en el año 2010 la inmensa mayoría de los fondos se destinaron a proyectos de inversión. Habría que estudiarlo más a fondo, pero esto puede estar directamente relacionado con la finalización del FAD y el Fondo de Concesión de Microcréditos y la creación en ese año del FONPRODE.

\section{INSTRUMENTOS DE LA COOPERACIÓN ESPAÑOLA Y EMPRESAS}

Además de los instrumentos financieros, como los antiguos FAD, FCM y actualmente FONPRODE -el cual está siendo remodelado legislativamente en estos días-, hay otros instrumentos de la cooperación española que permite la participación de empresas, como aparecen reflejados en las acciones subvencionadas en los datos proporcionados por el ministerio. Las dos principales son la Convocatoria Abierta y Permanente, que no impedía presentarse a las empresas privadas, además de la modalidad específica que convocó en el año 2011 (Resolución de 12 de mayo de 2011, de la Presidencia de la Agencia Española de Cooperación Internacional para el Desarrollo, por la que se convocan ayudas de convocatoria abierta y permanente para actividades de cooperación y ayuda al desarrollo, correspondiente al año 2011). El otro gran instrumento son las Alianzas Público Privadas, habiendo publicado recientemente la AECID un protocolo para empresas y organizaciones de la sociedad civil. Aunque anteriormente se han creado dichas alianzas con apoyo público, siendo la más conocida la APPD realizada en Acobamba, Perú, con la participación de Telefónica, BBVA y Grupo Santillana, este protocolo trata aspectos básicos como las características que deben de cumplir las entidades participantes en estas alianzas, y que probablemente ponga las bases para futurasacciones de la AECID 
en estamateria. Para las empresas presenta una serie de criterios excluyentes y criterios valorativos. Los excluyentes son los siguientes:

La AECID no podrá en ningún caso establecer alianzas con entidades que lleven a cabo las siguientes prácticas:

1. La fabricación, compra y venta de armas así como la financiación de actividades relacionadas.

2. La utilización directa o indirecta de trabajo infantil, de trabajo forzado o en régimen de esclavitud.

3. Prácticas antisindicales.

4. Actuaciones tipificadas como soborno y corrupción.

5. Actuaciones contra el medio ambiente.

A pesar de ser los mismos criterios que ya se introdujeron en la CAP línea empresarial, estos criterios son excesivamente generalistas, lo cual no quita que probablemente algunas de las empresas a las que se han concedido fondos no los cumplen. Habrá que esperar a ver cómo evolucionan los datos de la cooperación en los próximos años.

\section{TIPOLOGÍA DE LAS EMPRESAS}

Todavía resulta complicado realizar una tipología sistematizada de las empresas que reciben fondos públicos para cooperación al desarrollo debido a los problemas encontrados con las fuentes. Si no están todos los datos de las entidades que los reciben la sistematización no será posible, al menos de momento. Sin embargo, haciendo una revisión general sobre a qué empresas se han concedido fondos públicos se ha hecho una tipología muy funcional que se irá matizando a medida que se vayan consolidando los datos.

\section{EMPRESAS PÚBLICAS}

Aunque en principio las empresas públicas no constituían el centro de este estudio se han decidido incluir debido a que se encuentran presentes en prácticamente todos los niveles de la cooperación y sobre todo de la administración central éstas reciben una gran cantidad de fondos. La empresa pública estrella de la cooperación española es sin duda TRAGSA, que forma parte de un holding de empresas de la Sociedad Estatal de Participaciones Industriales. Sin embargo, no es la única, ya que nos encontramos también medios audiovisuales públicos, como TVE o TV3, que son subvencionadas fundamentalmente por la administración central y la Generalitat de Catalunya respectivamente, $\mathrm{o}$ incluso nos encontramos también con empresas públicas municipales, como el caso de Córdoba, con SADECO, una empresas municipal de saneamiento, o EMACSA, la empresa municipal de aguas. En este caso, estos fondos fueron concedidos por el ayuntamiento de Córdoba. 


\section{EMPRESAS PRIVADAS}

A pesar de que oficialmente la cooperación de la administración general del estado solo reconoce la Convocatoria Abierta y Permanente (CAP) del año 2011 (Resolución de 12 de mayo de 2011, de la Presidencia de la Agencia Española de Cooperación Internacional para el Desarrollo, por la que se convocan ayudas de convocatoria abierta y permanente para actividades de cooperación y ayuda al desarrollo, correspondiente al año 2011) específica para empresas como su único instrumento para subvencionar proyectos de empresas, y para la que se destinó 3 millones de euros, la realidad es que muchas empresas han recibido fondos a través de esta convocatoria aunque no fuera específica para empresas en años anteriores y posteriores. Por las características de esta convocatoria, el tipo de empresas que han recibido fondos son fundamentalmente consultoras y empresas de gestión cultural.

Sin embargo es fundamentalmente la cooperación financiera la que más dinero ha transferido hacia empresas. Primero a través de los conocidos créditos FAD, ya desaparecidos, aunque se han seguido pagando créditos ya concedidos hasta el año 2012, desconociendo los datos de la cooperación del 2013. Su estudio resulta complicado ya que aunque desde el ministerio publican las cuantías totales concedidas, cuando bajamos a los datos concretos oficialmente estos fondos se suelen transferir a entidades públicas de los países destino, que son los que reciben los fondos para transferirlos posteriormente a las empresas adjudicatarias. En este caso, estas empresas técnicamente no actuarían como agentes de cooperación. A pesar de esto, resulta fundamental reseñarlas por un lado por la gran crítica que supuso estos créditos al ser ayuda ligada y por otro lado porque han supuesto los mayores desembolsos de la cooperación española hacia las empresas privadas.

También tienen gran importancia los fondos concedidos para microcréditos, primero del ya extinto Fondo de Concesión de Microcréditos y posteriormente al FONPRODE. Las entidades que reciben estos fondos no tienen por qué ser empresas, en algunos casos son ONGD que dentro de sus proyectos tienen una parte dedicada a la microfinanciación. Sin embargo en otros casos son fondos de inversión o bancos de crédito que, claramente, son empresas privadas. Aquí nos encontramos un amplio espectro de entidades, pero hay fondos de inversión radicados en paraísos fiscales, como Angola o Panamá. Habría que aclarar que para acciones de cooperación, algunas de las subvenciones contienen un punto en el que derogan la ley de subvenciones para poder conceder fondos a entidades radicadas en paraísos fiscales, argumentando que muchos de ellos son países en vías de desarrollo. Esto permite que entidades financieras radicadas en estos países puedan acceder a estas subvenciones.

\section{FUNDACIONES DE EMPRESAS}

Este es probablemente el tema más controvertido de la relación entre la cooperación al desarrollo y el sector empresarial. Técnicamente las fundaciones no son empresas, pero es innegable la vinculación que existe entre las grandes transnacionales y sus propias fundaciones, creadas con capital de la empresa y que normalmente llevan el nombre de ésta. Esta ha sido la razón por la que las fundaciones de empresas han sido incluidas en el estudio, a pesar de oficialmente 
ser entidades sin ánimo de lucro. El caso más conocido de una fundación de empresas recibiendo fondos públicos fue el de la Fundación Repsol, que en septiembre de 2011 recibió una subvención de 150.000 euros para realizar un proyecto en Ecuador. Sin embargo ha habido casos anteriores y posteriores e involucrando a una gran variedad de fundaciones. Dos fundaciones que reciben fondos habitualmente son la Fundación del Real Madrid y la Fundación del FC Barcelona. Ambos reciben fondos casi en exclusividad para abrir y mantener escuelas de fútbol en países en vías de desarrollo. También han recibido fondos la Fundación SM, fundación de la editorial española, o incluso la Fundación Barceló, fundación del grupo hotelero.

Además de lo que entendemos por fundaciones de empresas, también existen fundaciones cuyo patronato o alto patronato está formado por empresas o al menos contiene alguna. Este es el caso del famoso Fondo de Ayuda contra la Drogadicción, en cuyo patronato están representadas todas las empresas del IBEX-35, todas las empresas de medios de comunicación, y otras. Esta fundación recibe fondos de la administración central y de algunas comunidades autónomas para realizar sus proyectos. El debate se abre porque técnicamente no es una empresa, pero la gran mayoría de transnacionales españolas están representadas ahí.

\section{ALIANZAS PÚBLICO PRIVADAS}

Las administraciones también destinan fondos a Alianzas Público Privadas ya consolidadas y de gran proyección internacional. Una entidad recurrente en el destino de fondos de la cooperación española es el IAVI, International AIDSVaccinelnitiative. Esta entidad es una alianza público privada en la que participa un buen número de las agencias de cooperación europeas, fundaciones como la de Bill y Melinda Gates y corporaciones privadas. Además de a otros partenariados público privados internacionales, como la Unión Internacional para la conservación de la naturaleza, UICN, también dedican fondos a uno de los partenariados público privados más conocido en la cooperación española, la Fundación Carolina. En su órgano de gobierno se encuentran tanto personalidades de las administraciones públicas como del sector privado con ánimo de lucro,

\section{OTRAS ENTIDADES}

Además de todas las entidades expuestas anteriormente, también nos encontramos más raramente con otras posibilidades de empresariado:

Organizaciones empresariales: fundamentalmente nos encontramos a dos, la CEOE y la CEA, la confederación de empresarios andaluces. Puesto que las organizaciones empresariales también están reconocidas como agentes de cooperación en principio no causa demasiados problemas al incluirlos en esta tipología.

Consorcios y similares: son instituciones de muy difícil catalogación. La mayoría de ellas son consorcios públicos en los que intervienen comunidades autónomas, ayuntamientos y ministerios. Son las "Casa América", "Casa Asia" etc. Aunque es cierto que tienen una parte muy importante de organización 
pública, la mayoría de ellas tienen en su patronato o alto patronato una gran representación de empresas transnacionales españolas de todo tipo. Este es otro caso en el que su propia definición y su introducción en las tipologías resulta complejo. Tomando el ejemplo de la Casa América, es un consorcio público que realiza multitud de actividades y que tiene en su consejo rector al Ministro de Asuntos Exteriores y Cooperación, al presidente de la Comunidad de Madrid y a la alcaldesa de Madrid. Sin embargo, en su alto patronato contiene empresas como Gas Natural, BBVA, Iberia, Grupo Santander, Telefónica y fundaciones de empresas como la Fundación Abertis, la Fundación ACS, entre otras.

Autónomos: aunque no es frecuente, entre los datos de la cooperación española nos encontramos de vez en cuando con personas que reciben fondos normalmente por servicios prestados, trabajo de consultoría y asesoría, y cuestiones similares. Estas personas individuales no pueden ser consideradas de otra manera, ya que están ofreciendo un servicio a cambio de una remuneración económica sin más apoyo organizativo.

\section{CONCLUSIONES}

Aunque todavía es pronto para poder sacarle pleno rendimiento a la base de datos que se está realizando para esta investigación, ya se pueden apreciar tendencias y extrapolar datos generales que nos empiezan a dar una idea de cómo ha sido la relación entre la administración pública y las empresas en los últimos años de vigencia del Plan Director 2009-2012. Es cierto que las empresas han estado representadas todos los años, aunque en manera desigual, con la particularidad del año 2010 donde se multiplicó prácticamente por diez los fondos destinados a estas entidades, probablemente vinculado a la aparición del FONPRODE y la desaparición de los órganos que le precedieron. Aparentemente, los resultados no ofrecen enormes cantidades destinadas a las empresas, o al menos no tantas como cabría esperar viendo el gran número de publicaciones al respecto. Los aspectos cualitativos de esta ayuda podría revelarnos muchas cosas, siendo la más importante descubrir cuál es el interés del sector empresarial en la cooperación al desarrollo.

A pesar de que los datos sobre orientación geográfica y tipología de la ayuda pueden ser anecdóticos, la base de datos permitiría analizar y clasificar las acciones prácticamente siguiendo cualquier criterio de búsqueda, en lo referente a las acciones realizadas por empresas. A su vez, esto permitiría el diseño de nuevas investigaciones. 


\title{
Companies in the Spanish government cooperation 2009-2012
}

\begin{abstract}
The research base is a database made primarily with the information provided by the Ministry of Foreign Affairs on the Official Development Assistance (ODA) during the years 2009-2012 Spanish. In this database it has been compiled exclusively the information on companies in a broad sense of the term that have received public money for projects considered ODA cooperation. With this information are extrapolating statistical data to get a clear picture of what has been the role of the business sector in the government's development cooperation during the term of the last Director of the Spanish Cooperation Plan 2009-2012, analyzing amounts, geotargeting, sectoral orientation and type of enterprises, among others.
\end{abstract}

KEYWORDS: Business cooperation. RSC. Corporate Social Responsibility. ODA. Official Development Assistance. 


\section{Empresas na cooperação governamental espanhola 2009-2012}

\section{SUMÁRIO}

A base de pesquisa é uma base de dados feita principalmente com a informação fornecida pelo Ministério dos Negócios Estrangeiros sobre a Assistência Oficial ao Desenvolvimento (ODA) espanhola durante os anos de 2009-2012. Neste banco de dados foi compilado exclusivamente a informação sobre as empresas num sentido lato do termo que receberam dinheiro público para projetos considerados cooperação ODA. Com esta informação está extrapolando dados estatísticos para obter uma imagem clara do que tem sido o papel do setor empresarial na cooperação para o desenvolvimento do governo durante a vigência do último Plano Director de Cooperação Espanhola 20092012, analisando quantias, segmentação geográfica, orientação sectorial e tipo de empresa, entre outros.

Palavras-chave: Cooperação empresarial. RSC. Responsabilidade social corporativa. ODA. Ajuda Pública ao Desenvolvimento. 


\section{REFERENCIAS}

ROMERO, M. y RAMIRO, P.Pobreza 2.0. Empresas, estados y ONGD ante la privatización de la cooperación al desarrollo. Ed. Icaria, Barcelona. 2012.

PINTADO MAROTO, E.El sector empresarial y la cooperación gubernamental al desarrollo en España, TFM del Master Oficial de Cooperación de la Universidad de Valladolid. 2012.

FRESNILLO, I. Nuevo rumbo (empresarial) para la cooperación internacional. El caso de del nuevo Fondo para el Desarrollo (FONPRODE). Boletín ODG, núm. Junio 2011. 2011.

VAZQUEZ, O. Las APPD y la necesidad de contar con sistemas de elegibilidad. Criterios de elegibilidad», Boletín ODG, núm. Mayo 2011, pp. 1-2. 2011.

MAEC. SECI. (2009) III Plan Director de la Cooperación Española 2009-2012.

MAEC. SECI. (2009) Seguimiento del PACI 2009

MAEC. SECI. (2009) Volcado del Seguimiento PACI 2009

MAEC. SECI. (2010) Seguimiento del PACI 2010

MAEC. SECI. (2010) Volcado del Seguimiento PACI 2010

MAEC. SECI.Estrategia de crecimiento económico y promoción del tejido empresarial, DGPOLDE. 2011.

MAEC. SECI (2011). Seguimiento del PACI 2011

MAEC. SECI. (2011) Volcado del Seguimiento PACI 2011

MAEC. SECI (2012). Seguimiento del PACI 2012

MAEC. SECI. (2012) Volcado del Seguimiento PACI 2012 
Recebido: 08 abr. 2016

Aprovado: 13 jun. 2016

DOI: $10.3895 /$ rbpd.v5n2.4496

Como citar: MAROTO, E. P. Empresas en la cooperación gubernamental española 2009-2012. R. bras.

Planej. Desenv., Curitiba, v. 5, n. 2, p. 255-269, mai./ago. 2016. Disponível em:

$<$ https://periodicos.utfpr.edu.br/rbpd>. Acesso em: XXX.

Correspondência:

Elisa Pintado Maroto

C/Plaza de Santa Cruz, 8, 47002 Valladolid, España.

Direito autoral: Este artigo está licenciado sob os termos da Licença CreativeCommons-Atribuição 4.0 Internacional.

\section{(c) (1)}

